\title{
On the State Constraint Problem for Differential Games
}

\section{SHIGEAKI KOIKE}

Abstract. The state constraint problem for differential games is treated. The representation formulas for viscosity solutions of the problem are given. The uniqueness of the solutions is shown under natural boundary conditions, which are derived from dynamic programming principle.

1. Introduction. In this paper we are concerned with the state-space constraint (SC in short) problems in differential games. We will discuss the following fully nonlinear first-order PDEs of min-max and max-min types:

$$
\min _{b \in B} \max _{a \in A}\{\lambda u(x)-\langle g(x, a, b), D u(x)\rangle-f(x, a, b)\}=0
$$

and

$$
\max _{a \in A} \min _{b \in B}\{\lambda u(x)-\langle g(x, a, b), D u(x)\rangle-f(x, a, b)\}=0
$$

for $x \in \Omega$, where $\Omega \subset \mathbb{R}^{n}$ is an open bounded set, $A$ and $B$ are compact sets in $\mathbb{R}^{N}$ for some $N \in \mathbb{N}, f$ and $g$ are given continuous real-valued and $\mathbb{R}^{n}$-valued functions, respectively, on $\bar{\Omega} \times A \times B$, and $\lambda$ is a positive constant. We occasionally use the same notation $f$ and $g$ for their continuous extensions to $\mathbb{R}^{n} \times A \times B$.

The SC problem was first treated via the viscosity solution approach by Soner in [14] for deterministic optimal control problems, where the associated value functions satisfy first order PDEs of Bellman type. He proposed an appropriate boundary condition for the PDEs under which the value function is the unique continuous viscosity solution. Under this setting, the result has been extended to second order degenerate elliptic PDEs and "monotone" systems of those. For the developement in this direction, we refer to [1], [2] and [12].

Recently, Katsoulakis in [10] pointed out that we only need a "non-tangential semicontinuity" assumption for solutions to show the uniqueness of viscosity 
solutions for second order degenerate elliptic PDEs under Soner's setting. He also showed the existence of viscosity solutions having such semicontinuity. See also [11] for a generalization of this result to monotone systems.

More recently, for first order Bellman equations, Ishii and Koike in [8] have proposed a new boundary condition, which is naturally derived from the SC requirement. In fact, in [8], it was shown that the value function is the unique viscosity solution among possibly discontinuous viscosity solutions under the new boundary condition.

On the other hand, from the point of view of applications, it is important to study differential game problems under the SC requirement. It is well-known that the associated value functions satisfy $(1.1 \pm)$ in the viscosity sense in $\Omega$. Following [8], under the SC requirement, we will derive the appropriate boundary conditions for $(1.1 \pm)$ which the value functions satisfy. It will turn out that the value functions are the unique viscosity solutions of the boundary value problems.

Our first aim here is to present the definition of value functions for the SC problem of differential games in a reasonable way. For this purpose, we will introduce the admissible strategies for the SC problem for differential games so that the dynamic programming principle (DPP in short) holds and, in the sequel, the value functions are viscosity solutions of our SC problems.

We will show the uniqueness of viscosity solutions without the continuity assumption for the solutions as shown for PDEs of Bellman type in [8]. To this end, we will employ some idea initiated by Dupuis and Ishii in [3].

We refer to [1], [4], [6], [13], [15] and their references for the related problems concerning Isaacs equations via the viscosity solution approach.

We also refer to [2] for the standard notations in the theory of viscosity solutions.

This paper is basically organized in the style of Evans and Souganidis in [6]: Section 2 is devoted to define our lower and upper value functions for the SC problems associated with $(1.1-)$ and $(1.1+)$, respectively. We show that the DPP holds for these values functions in Section 3. In Section 4 we present some stability properties for the Hamiltonians. Then, employing these results, we verify that the value functions are indeed viscosity solutions in Section 5. In Section 6 we present our comparison principle for viscosity solutions of our $\mathrm{SC}$ problems. In the final section we give a proof of a lemma concerning the construction of test functions.

2. Lower and upper values. We shall define the lower and upper values for the SC problem associated with $(1.1-)$ and $(1.1+)$.

We suppose the following assumptions in what follows: 


$$
\left\{\begin{array}{l}
\text { There is a modulus of continuity } \omega_{0} \text { such that } \\
\text { (1) }|g(x, a, b)-g(x, \hat{a}, \hat{b})| \leq \omega_{0}(|a-\hat{a}|+|b-\hat{b}|), \\
\text { (2) }|f(x, a, b)-f(\hat{x}, \hat{a}, \hat{b})| \leq \omega_{0}(|x-\hat{x}|+|a-\hat{a}|+|b-\hat{b}|) \text { for all } x, \\
\\
\quad \hat{x} \in \bar{\Omega}, a, \hat{a} \in A, b, \hat{b} \in B, \text { and } \\
\text { (3) } \sup _{(a, b) \in A \times B}\left\{\|f(\cdot, a, b)\|_{C(\bar{\Omega})}+\|g(\cdot, a, b)\|_{C^{0,1}(\bar{\Omega})}\right\}<\infty .
\end{array}\right.
$$

We denote by $\mathcal{A}$ and $\mathcal{B}$, respectively, the sets of controls by the players I and II;

$$
\mathcal{A}=\{\alpha:[0, \infty) \rightarrow A \mid \alpha: \text { measurable }\}
$$

and

$$
\mathcal{B}=\{\beta:[0, \infty) \rightarrow B \mid \beta: \text { measurable }\} .
$$

Throughout this paper, for $x \in \bar{\Omega}, \alpha \in \mathcal{A}$ and $\beta \in \mathcal{B}, X(\cdot ; x, \alpha, \beta)$ denotes the unique solution of the following:

$$
\left\{\begin{array}{c}
\frac{d X}{d t}(t)=g(X(t), \alpha(t), \beta(t)) \quad \text { for } t>0 \\
X(0)=x .
\end{array}\right.
$$

We will occasionally identify $a \in A$ and $b \in B$ with $\alpha(\cdot) \equiv a \in \mathcal{A}$ and $\beta(\cdot) \equiv$ $b \in \mathcal{B}$, respectively. For instance, we will often use the notations $X(t ; x, a, \beta) \equiv$ $X(t ; x, \alpha, \beta)$ for $a \in A$ by setting $\alpha(\cdot) \equiv a$, etc.

Roughly speaking, our SC problem is as follows: For each $x \in \bar{\Omega}$, the players I and II, respectively, "minimize and maximize" a functional $J(x, \alpha, \beta)$ over $\alpha \in \mathcal{A}$ and $\beta \in \mathcal{B}$ for which $X(t ; x, \alpha, \beta)$ stays in $\bar{\Omega}$ for any time $t \geq 0$. Then, we will derive functions depending on the $x$, which we will call the lower and upper value functions. Our main discussion is to characterize the PDE (particularly its boundary condition) that the value functions satisfy in the sense of viscosity solutions.

We shall define the set of admissible pairs of controls for our SC problems of $(1.1 \pm)$ at $x \in \bar{\Omega}$ and up to a time $s \in(0, \infty]$ :

$$
A D_{s}(x)=\{(\alpha, \beta) \in \mathcal{A} \times \mathcal{B} \mid X(t ; x, \alpha, \beta) \in \bar{\Omega} \text { for } t \in[0, s]\} .
$$

We also define the sets of admissible controls at $x \in \bar{\Omega}$ and up to $s \in(0, \infty]$ for the players I and II, respectively:

$$
\mathcal{A}_{s}(x)=\left\{\alpha \in \mathcal{A} \mid \text { There is } \beta \in \mathcal{B} \text { such that }(\alpha, \beta) \in A D_{s}(x)\right\},
$$


and

$$
\mathcal{B}_{s}(x)=\left\{\beta \in \mathcal{B} \mid \text { There is } \alpha \in \mathcal{A} \text { such that }(\alpha, \beta) \in A D_{s}(x)\right\} .
$$

In what follows, we suppose the following:

$$
\mathcal{A}_{\infty}(x) \neq \varnothing \text { (or quivalently } \mathcal{B}_{\infty}(x) \neq \varnothing \text { ) for all } x \in \bar{\Omega} \text {. }
$$

Next, we define the sets of strategies up to time $s \in(0, \infty]$ by II and I, respectively:

$$
\Gamma_{s}=\left\{\begin{array}{l|l}
\gamma: \mathcal{A} \rightarrow \mathcal{B} & \begin{array}{l}
\text { For all } t \in(0, s], \text { if } \alpha=\hat{\alpha} \text { a. e. in }(0, t) \\
\text { for } \alpha, \hat{\alpha} \in \mathcal{A}, \text { then } \gamma[\alpha]=\gamma[\hat{\alpha}] \text { a. e. in } \\
(0, t) .
\end{array}
\end{array}\right\}
$$

and

$$
\Delta_{s}=\left\{\begin{array}{l|l}
\delta: \mathcal{B} \rightarrow \mathcal{A} & \begin{array}{l}
\text { For all } t \in(0, s], \text { if } \beta=\hat{\beta} \text { a. e. in }(0, t) \\
\text { for } \beta, \hat{\beta} \in \mathcal{B}, \text { then } \delta[\beta]=\delta[\hat{\beta}] \text { a. e. in } \\
(0, t) .
\end{array}
\end{array}\right\}
$$

In order to treat our SC problems, we introduce the sets, $\Gamma_{s}(x)$ and $\Delta_{s}(x)$, which consist of all admissible strategies for $x \in \bar{\Omega}$ and $s \in(0, \infty]$ :

$$
\Gamma_{s}(x)=\left\{\gamma \in \Gamma_{s} \mid(\alpha, \gamma[\alpha]) \in A D_{s}(x) \text { for all } \alpha \in \mathcal{A}_{s}(x)\right\},
$$

and

$$
\Delta_{s}(x)=\left\{\delta \in \Delta_{s} \mid(\delta[\beta], \beta) \in A D_{s}(x) \text { for all } \beta \in \mathcal{B}_{s}(x)\right\} .
$$

We remark that, for each $x \in \Omega$, according to $(A 1)$, there is $s>0$ such that

$$
\mathcal{A}_{s}(x)=\mathcal{A} \text { and } \mathcal{B}_{s}(x)=\mathcal{B} \text {. }
$$

We then define the pay-off functional at $x \in \bar{\Omega}$ associated with (1.1 \pm ) up to a time $s \in(0, \infty]$ for each $(\alpha, \beta) \in \mathcal{A} \times \mathcal{B}$ :

$$
J^{s}(x, \alpha, \beta)=\int_{0}^{s} e^{-\lambda t} f(X(t ; x, \alpha, \beta), \alpha(t), \beta(t)) d t .
$$

Now we define the lower value function $V$ and the upper value function $U$, respectively, on $\bar{\Omega}$ in the following manner:

$$
V(x) \equiv \inf _{\delta \in \Delta_{\infty}(x)} \sup _{\beta \in \mathcal{B}_{\infty}(x)} J^{\infty}(x, \delta[\beta], \beta),
$$


and

$$
U(x) \equiv \sup _{\gamma \in \Gamma_{\infty}(x)} \inf _{\alpha \in \mathcal{A}_{\infty}(x)} J^{\infty}(x, \alpha, \gamma[\alpha]) .
$$

We remark that the value function $U$ coincides with that for the control problem in [8] whenever $B$ consists of one control parameter.

From the definition, we immediately obtain the following bound.

Proposition 2.1 Assume $(A 1)$ and $(A 2)$. Then, we have

$$
\sup _{x \in \bar{\Omega}}|V(x)|<\infty \text { and } \sup _{x \in \bar{\Omega}}|U(x)|<\infty .
$$

3. Dynamic programming principle. In this section we present the DPP for our value functions.

Theorem 3.1 (cf. [6]) Assume (A1) and (A2). Let $V$ and $U$ be the lower and upper value functions in the above. Fix any $x \in \bar{\Omega}$ and $s>0$. Then, we have

$$
V(x)=\inf _{\delta \in \Delta_{s}(x)} \sup _{\beta \in \mathcal{B}_{s}(x)}\left\{J^{s}(x, \delta[\beta], \beta)+e^{-\lambda s} V(X(s ; x, \delta[\beta], \beta))\right\}
$$

and

$$
U(x)=\sup _{\gamma \in \Gamma_{s}(x)} \inf _{\alpha \in \mathcal{A}_{s}(x)}\left\{J^{s}(x, \alpha, \gamma[\alpha])+e^{-\lambda s} U(X(s ; x, \alpha, \gamma[\alpha]))\right\}
$$

Proof. We give the details of a proof of (3.1) for the reader's convenience, although the proof is similar to that of [6]. However, we need some careful observation with respect to what follows, because we deal with admissible controls and strategies.

Fix $s>0$. We define the right hand side of (3.1) by $W(x)$;

$$
W(x)=\inf _{\delta \in \Delta_{s}(x)} \sup _{\beta \in \mathcal{B}_{s}(x)}\left\{J^{s}(x, \delta[\beta], \beta)+e^{-\lambda s} V(X(s ; x, \delta[\beta], \beta))\right\} .
$$

We only show the case when $x \in \partial \Omega$ since the other case can be proved similarly.

To show (3.1), we will prove $V(x) \geq W(x)$ and $V(x) \leq W(x)$.

For any small $\varepsilon>0$, there is $\delta_{0} \in \Delta_{\infty}(x)$ such that

$$
V(x)+\varepsilon \geq \sup _{\beta \in \mathcal{B}_{\infty}(x)} J^{\infty}\left(x, \delta_{0}[\beta], \beta\right) .
$$


Fix any $\beta_{0} \in \mathcal{B}_{s}(x)$ and define $\hat{x}=X\left(s ; x, \delta_{0}\left[\beta_{0}\right], \beta_{0}\right)$.

We introduce a mapping $T: \mathcal{B} \rightarrow \mathcal{B}$ in the following way: For $\beta \in \mathcal{B}$,

$$
T \beta(t)= \begin{cases}\beta_{0}(t) & \text { for } t \in[0, s] \\ \beta(t-s) & \text { for } t>s .\end{cases}
$$

We set $\hat{\delta}_{0} \in \Delta_{\infty}(\hat{x})$ as follows: For any $\beta \in \mathcal{B}$,

$$
\hat{\delta}_{0}[\beta](t)=\delta_{0}[T \beta](t+s) \text { for } t \geq 0 .
$$

Choose any $\beta \in \mathcal{B}_{\infty}(\hat{x})$. Noting $T \beta \in \mathcal{B}_{\infty}(x)$, we substitute $T \beta$ in (3.3) to obtain

$$
\begin{aligned}
V(x)+\varepsilon \geq J^{s}\left(x, \delta_{0}\left[\beta_{0}\right]\right. & \left., \beta_{0}\right) \\
& +\int_{s}^{\infty} e^{-\lambda t} f\left(X\left(t ; x, \delta_{0}[T \beta], T \beta\right), \delta_{0}[T \beta](t), T \beta(t)\right) d t .
\end{aligned}
$$

It is easy to verify that the second term of the right hand side of the inequality above can be replaced by $e^{-\lambda s} J^{\infty}\left(\hat{x}, \hat{\delta}_{0}[\beta], \beta\right)$.

Since this holds for any $\beta \in \mathcal{B}_{\infty}(\hat{x})$, we have

$$
\begin{aligned}
V(x)+\varepsilon & \geq J^{s}\left(x, \delta_{0}\left[\beta_{0}\right], \beta_{0}\right)+e^{-\lambda s} \sup _{\beta \in \mathcal{B}_{\infty}(\hat{x})} J^{\infty}\left(\hat{x}, \hat{\delta}_{0}[\beta], \beta\right) \\
& \geq J^{s}\left(x, \delta_{0}\left[\beta_{0}\right], \beta_{0}\right)+e^{-\lambda s} V(\hat{x}) .
\end{aligned}
$$

Hence, taking the supremum over all $\beta_{0} \in \mathcal{B}_{s}(x)$ in (3.4), we have

$$
V(x)+\varepsilon \geq W(x) .
$$

Next, we shall verify $V(x) \leq W(x)$. Fix any $\varepsilon>0$ again. Then, there is $\delta_{0} \in \Delta_{s}(x)$ such that

$$
W(x)+\varepsilon \geq \sup _{\beta \in \mathcal{B}_{s}(x)}\left\{J^{s}\left(x, \delta_{0}[\beta], \beta\right)+e^{-\lambda s} V\left(X\left(s ; x, \delta_{0}[\beta], \beta\right)\right)\right\} .
$$

On the other hand, for each $y \in \bar{\Omega}$, we choose $\delta_{y} \in \Delta_{\infty}(y)$ so that

$$
V(y)+\varepsilon \geq \sup _{\tilde{\beta} \in \mathcal{B}_{\infty}(y)} J^{\infty}\left(y, \delta_{y}[\tilde{\beta}], \tilde{\beta}\right) .
$$

We shall define a mapping $S: \mathcal{B} \rightarrow \mathcal{B}$ in the following way: For $\tilde{\beta} \in \mathcal{B}$,

$$
S \tilde{\beta}(t)=\tilde{\beta}(t+s) \text { for } t \geq 0 .
$$


Now, we shall construct $\hat{\delta} \in \Delta_{\infty}(x)$. To this end, we fix any $\beta \in \mathcal{B}_{\infty}(x)$ and set $x_{\beta}=X\left(s ; x, \delta_{0}[\beta], \beta\right)$. We choose $\delta_{x_{\beta}} \in \Delta_{\infty}\left(x_{\beta}\right)$ satisfying (3.6) with $y=x_{\beta}$.

For $\beta \in \mathcal{B}_{\infty}(x)$, we define the mapping $\hat{\delta} \in \Delta_{\infty}(x)$ by

$$
\hat{\delta}[\beta](t) \equiv \alpha(t)= \begin{cases}\delta_{0}[\beta](t) & \text { for } t \in[0, s] \\ \delta_{x_{\beta}}[S \beta](t-s) & \text { for } t>s .\end{cases}
$$

Noting $S \beta \in \mathcal{B}_{\infty}\left(x_{\beta}\right)$, we substitute $S \beta$ in (3.6) with $y=x_{\beta}$ to obtain

$$
V\left(x_{\beta}\right)+\varepsilon \geq J^{\infty}\left(x_{\beta}, \delta_{x_{\beta}}[S \beta], S \beta\right) .
$$

Hence, substituting this in (3.5), we have

$$
W(x)+2 \varepsilon \geq J^{s}\left(x, \delta_{0}[\beta], \beta\right)+e^{-\lambda s} J^{\infty}\left(x_{\beta}, \delta_{x_{\beta}}[S \beta], S \beta\right) .
$$

Since $X\left(t ; x_{\beta}, \delta_{x_{\beta}}[S \beta], S \beta\right)=X(t+s ; x, \hat{\delta}[\beta], \beta)$ holds for $t \geq 0$, we can rewrite (3.7) into the following:

$$
W(x)+2 \varepsilon \geq J^{\infty}(x, \hat{\delta}[\beta], \beta) .
$$

Taking the supremum over all $\beta \in \mathcal{B}_{\infty}(x)$, we have $W(x)+2 \varepsilon \geq V(x)$.

4. Stability properties. We shall employ the following notation for simplicity:

$$
H(x, r, p ; a, b)=\lambda r-\langle g(x, a, b), p\rangle-f(x, a, b)
$$

for $(x, r, p, a, b) \in \bar{\Omega} \times \mathbb{R} \times \mathbb{R}^{n} \times A \times B$.

We then define the lower and upper Hamiltonians, respectively, as follows:

$$
\underline{H}(x, r, p)=\min _{b \in B} \max _{a \in A} H(x, r, p ; a, b)
$$

and

$$
\bar{H}(x, r, p)=\max _{a \in A} \min _{b \in B} H(x, r, p ; a, b)
$$

for $(x, r, p) \in \bar{\Omega} \times \mathbb{R} \times \mathbb{R}^{n}$. Note that, by $(A 1), \underline{H}$ and $\bar{H}$ are continuous in $\bar{\Omega} \times \mathbb{R} \times \mathbb{R}^{n}$. 
For each $(x, a, b) \in \bar{\Omega} \times A \times B$, we define the following subsets of $A$ and $B$ :

$$
A(x, b)=\left\{\begin{array}{l|l}
a \in A & \begin{array}{l}
\text { There is } r>0 \text { such that } X(t ; y, a, b) \in \\
\bar{\Omega} \text { for } t \in[0, r] \text { provided } y \in \bar{\Omega} \cap B_{r}(x) .
\end{array}
\end{array}\right\}
$$

and

$$
B(x, a)=\left\{\begin{array}{l|l}
b \in B & \begin{array}{l}
\text { There is } r>0 \text { such that } X(t ; y, a, b) \in \\
\bar{\Omega} \text { for } t \in[0, r] \text { provided } y \in \bar{\Omega} \cap B_{r}(x) .
\end{array}
\end{array}\right\}
$$

Here and later, $B_{r}(x)$ denotes the standard $n$-dimensional closed ball with the center $x \in \mathbb{R}^{n}$ and the radius $r>0$.

Notice that, if $x \in \Omega$, then $A(x, b)=A$ and $B(x, a)=B$ for any $b \in B$ and $a \in A$.

We shall explain the reason why we will use $A(x, b)$ in the SC boundary condition for $(1.1-)$, for instance. Indeed, since the player II first selects a control in $\mathcal{B}_{\infty}(x)$ (say $b \in B$ ) to maximize the pay-off functional for the lower value function $V$, the player $\mathrm{I}$ is only allowed to choose a control (say $a \in A$ ) such that $(a, b) \in A D_{s}(x)$ for a small $s>0$. Since the boundary condition will be determined by the DPP, it seems reasonable that the pairs $(a, b) \in A \times B$ with $(a, b) \in A D_{s}(x)$ (for some $s>0$ ) appear in our boundary condition. We remark that, in our argument, the pair $(a, b)$ with $a \in A(x, b)$ need to be admissible at any point close to $x$. We refer to [8] for the admissible control set associated with the SC problem for optimal control.

We will need to suppose not only $A(x, b) \neq \varnothing$ (for $x \in \bar{\Omega}$ and $b \in B$ ) but also the requirement that, if $a \in A(x, b)$ and $\beta \in \mathcal{B}$ is close enough to $b$, then $(a, \beta)$ is an admissible pair;

$$
\left\{\begin{array}{l}
\text { There are } r, s>0 \text { such that, if } b \in B \text { and } \beta \in \mathcal{B} \text { satisfy } \\
|\beta(t)-b|<r \text { for a. a. } t \in[0, s], \text { and } x \in \partial \Omega, \text { then } A(x, b) \neq \\
\varnothing, \text { and } X(t ; x, a, \beta) \in \bar{\Omega} \text { for } t \in[0, s] \text { and } a \in A(x, b),
\end{array}\right.
$$

and

$$
\left\{\begin{array}{l}
\text { There are } r, s>0 \text { such that, if } a \in A \text { and } \alpha \in \mathcal{A} \text { satisfy } \\
|\alpha(t)-a|<r \text { for a. a. } t \in[0, s], \text { and } x \in \partial \Omega \text {, then } \\
B(x, a) \neq \varnothing, \text { and } X(t ; x, \alpha, b) \in \bar{\Omega} \text { for } t \in[0, s] \text { and } b \in \\
B(x, a) .
\end{array}\right.
$$

We remark that $(A 3)$ (resp., $\left(A 3^{\prime}\right)$ ) holds whenever $B$ (resp., $A$ ) is a finite set and $A(x, b) \neq \varnothing$ (resp., $B(x, a) \neq \varnothing$ ) for $b \in B$ (resp., $a \in A$ ).

Under these hypotheses, we define the upper and lower inner Hamiltonians $H_{\mp}: \bar{\Omega} \times \mathbb{R} \times \mathbb{R}^{n} \rightarrow \mathbb{R}$, respectively, by

$$
H_{-}(x, r, p)=\inf _{b \in B} \sup _{a \in A(x, b)} H(x, r, p ; a, b),
$$


and

$$
H_{+}(x, r, p)=\sup _{a \in A} \inf _{b \in B(x, a)} H(x, r, p ; a, b) .
$$

In order to verify that $V$ is a viscosity solution for the SC problem of $(1.1-)$ (see the definition in the next section), we will need the following "stability" properties for the Hamiltonians:

Lemma 4.1 (cf. [6]) Assume (A1).

(1) Let $(A 2)$ also hold. For any $\theta>0$ and $\phi \in C^{1}(\bar{\Omega})$, there exists $s>0$ such that, if

$$
\underline{H}(x, \phi(x), D \phi(x)) \leq-\theta
$$

holds for some $x \in \bar{\Omega}$, then there is $\beta_{0} \in \mathcal{B}_{s}(x)$ such that

$$
\int_{0}^{t} e^{-\lambda r} H\left(X_{\delta}(r), \phi\left(X_{\delta}(r)\right), D \phi\left(X_{\delta}(r)\right) ; \delta\left[\beta_{0}\right](r), \beta_{0}(r)\right) d r \leq-\frac{\theta}{2 \lambda}\left(1-e^{-\lambda t}\right)
$$

for $t \in(0, s]$ and $\delta \in \Delta_{t}(x)$, where $X_{\delta}(r)=X\left(r ; x, \delta\left[\beta_{0}\right], \beta_{0}\right)$.

(2) Let (A3) also hold. For any $\theta>0$ and $\phi \in C^{1}(\bar{\Omega})$, there exists $s>0$ such that, if

$$
H_{-}(x, \phi(x), D \phi(x)) \geq \theta
$$

holds for some $x \in \bar{\Omega}$, then there is $\delta_{0} \in \Delta_{s}(x)$ such that

$$
\begin{aligned}
& \int_{0}^{t} e^{-\lambda r} H\left(X_{\beta}(r), \phi\left(X_{\beta}(r)\right), D \phi\left(X_{\beta}(r)\right) ; \delta_{0}[\beta](r), \beta(r)\right) d r \geq \frac{\theta}{2 \lambda}\left(1-e^{-\lambda t}\right) \\
& \text { for } t \in(0, s] \text { and } \beta \in \mathcal{B}_{t}(x), \text { where } X_{\beta}(r)=X\left(r ; x, \delta_{0}[\beta], \beta\right) .
\end{aligned}
$$

Proof. (i) From (4.1), there is $b_{0} \in B$ such that

$$
H\left(x, \phi(x), D \phi(x) ; a, b_{0}\right) \leq-\theta
$$

for all $a \in A$.

In view of $(A 1)-(i i)$, (iii), we find $r>0$ satisfying that

$$
H\left(y, \phi(y), D \phi(y) ; a, b_{0}\right) \leq-\frac{\theta}{2}
$$


for $y \in B_{r}(x)$ and $a \in A$. By (A1)-(iii) again, we find $s>0$ independent of $x$ such that

$$
X\left(t ; x, \alpha, b_{0}\right) \in B_{r}(x)
$$

for any $\alpha \in \mathcal{A}$ and $t \in(0, s]$, where $r>0$ is a constant in (A3) for the $x$.

Thus, fixing $t \in(0, s]$ and then, choosing any $\delta \in \Delta_{t}(x)$, we have

$$
H\left(X_{\delta}(r), \phi\left(X_{\delta}(r)\right), D \phi\left(X_{\delta}(r)\right) ; \delta\left[b_{0}\right](r), b_{0}\right) \leq-\frac{\theta}{2}
$$

for $r \in(0, t]$, where $X_{\delta}(r) \equiv X\left(r ; x, \delta\left[b_{0}\right], b_{0}\right)$.

Multiply this inequality with $e^{-\lambda r}$ and then integrate that with respect to $r$ over $(0, t)$. Then, taking $\beta_{0}(\cdot)=b_{0}$, we conclude the proof.

(ii) First, for any $b \in B$, from the definition of $A(x, b)$, there is $a_{b} \in A(x, b)$ such that

$$
H\left(x, \phi(x), D \phi(x) ; a_{b}, b\right) \geq \theta .
$$

We take a constant $r>0$ in $(A 3)$. In view of $(A 1)$, we find $r_{b} \in(0, r)$ such that

$$
H\left(y, \phi(y), D \phi(y) ; a_{b}, \tilde{b}\right) \geq \frac{1}{2} \theta
$$

for $y \in B_{r_{b}}(x)$ and $\tilde{b} \in B$ with $|\tilde{b}-b|<r_{b}$.

Then, from the compactness of $B$, there are $\left\{r_{i} \in(0, r) \mid i=1,2, \ldots, m\right\}$ and a subset of $A \times B$,

$$
\left\{\left(\hat{a}_{i}, \hat{b}_{i}\right) \in A \times B \mid \hat{a}_{i} \in A\left(x, \hat{b}_{i}\right) \text { for } i=1, \ldots, m\right\},
$$

for some integer $m$, such that

$$
H\left(y, \phi(y), D \phi(y) ; \hat{a}_{i}, b\right) \geq \frac{\theta}{2} \text { provided } b \in B_{i} \text { and } y \in B_{r_{i}}(x),
$$

for all $i \in\{1,2, \ldots, m\}$, and $B=\bigcup_{i=1}^{m} B_{i}$, where $B_{i}=\left\{b \in B|| b-\hat{b}_{i} \mid<r_{i}\right\}$. Set $\hat{B}_{1}=B_{1}$ and $\hat{B}_{k}=B_{k}-\bigcup_{i=1}^{k-1} B_{i}$ for $2 \leq k \leq m$.

We shall define $\delta_{0}[\beta]$ by the following:

$$
\delta_{0}[\beta](t) \equiv a_{i} \text { provided } \beta(t) \in \hat{B}_{i} .
$$

Now, we claim $\delta_{0} \in \Delta_{s}(x)$ for some small $s>0$; For any fixed $\beta \in \mathcal{B}_{s}(x)$, we show that $X(t) \equiv X\left(t ; x, \delta_{0}[\beta], \beta\right) \in \bar{\Omega}$ for $t \in[0, s]$. Let us suppose the contrary. Assume that there is $t_{0} \in[0, s)$ such that

$$
X\left(t_{0}\right) \in \partial \Omega \text { and } X(t) \notin \bar{\Omega} \text { for } t \in\left(t_{0}, t_{0}+\sigma\right)
$$


for some small $\sigma>0$. We may suppose $t_{0}=0$.

For this $\beta \in \mathcal{B}_{s}(x)$, we define approximate functions $\beta^{\varepsilon} \in \mathcal{B}$ for $\varepsilon>0$ in the following manner: Set $T_{i}=\left\{t \in[0, s] \mid \beta(t) \in \hat{B}_{i}\right\}$ for $i=1,2, \ldots, m$. For each $\varepsilon>0$, we first choose open sets $\left\{J_{i}\right\}_{i=1}^{m}$ such that

$$
T_{i} \subset J_{i} \text { and }\left|J_{i}-T_{i}\right|<\varepsilon \text {. }
$$

We may suppose that $[0, \sigma) \subset J_{1}$. Set $\hat{J}_{1}=J_{1}$. We then inductively define $\hat{J}_{i} \equiv J_{i}-\bigcup_{\ell=1}^{i-1} \hat{J}_{l}$ for $2 \leq i \leq m$. Thus, we set

$$
\beta^{\varepsilon}(t)= \begin{cases}\beta(t) & \text { for } t \in \hat{J}_{i} \cap T_{i} \\ \hat{b}_{i} & \text { for } t \in \hat{J}_{i} \backslash T_{i}\end{cases}
$$

We easily see that

$$
\int_{0}^{s}\left|\beta^{\varepsilon}(t)-\beta(t)\right| d t \leq 2 m \varepsilon \max _{b \in B}|b|,
$$

and

$$
\int_{0}^{s}\left|\delta_{0}\left[\beta^{\varepsilon}\right](t)-\delta_{0}[\beta](t)\right| d t \leq 2 m \varepsilon \max _{a \in A}|a| .
$$

Setting $X_{\varepsilon}(t)=X\left(t ; x, \delta_{0}\left[\beta^{\varepsilon}\right], \beta^{\varepsilon}\right)$, we shall show that

$$
\lim _{\varepsilon \rightarrow 0} \max _{t \in[0, s]}\left|X_{\varepsilon}(t)-X(t)\right|=0
$$

for some $s>0$.

We fix $s \in(0, \min \{r, 1 /(2 L)\})$, where $L \geq \sup _{(a, b) \in A \times B}\|D g(\cdot, a, b)\|_{L^{\infty}(\Omega)}$. From the definition, we see that, for $t \in[0, s]$,

$$
\left|X_{\varepsilon}(t)-X(t)\right| \leq \int_{0}^{s}\left|g\left(X_{\varepsilon}(r), \delta_{0}\left[\beta^{\varepsilon}\right](r), \beta^{\varepsilon}(r)\right)-g\left(X(r), \delta_{0}[\beta](r), \beta(r)\right)\right| d r .
$$

By $(A 1)-(\mathrm{i})$, (iii), the right hand side of the inequality above is estimated from above by the following:

$$
\int_{0}^{s} \omega_{0}\left(\left|\delta_{0}\left[\beta^{\varepsilon}\right](r)-\delta_{0}[\beta](r)\right|+\left|\beta^{\varepsilon}(r)-\beta(r)\right|\right) d r+s L \max _{t \in[0, s]}\left|X_{\varepsilon}(t)-X(t)\right| .
$$

Since we may suppose that the modulus of continuity $\omega_{0}$ is concave, by the choice 
of $s>0$, we have

$$
\max _{t \in[0, s]}\left|X_{\varepsilon}(t)-X(t)\right| \leq 2 s \omega_{0}\left(\frac{1}{s} \int_{0}^{s}\left(\left|\beta^{\varepsilon}(r)-\beta(r)\right|+\left|\delta_{0}\left[\beta^{\varepsilon}\right](r)-\delta_{0}[\beta](r)\right|\right) d r\right) .
$$

This together with (4.4) and (4.5) yields (4.6).

The uniform convergence (4.6) and (4.3) implies that there is $t_{\varepsilon} \in[0, \sigma)$ such that $X_{\varepsilon}\left(t_{\varepsilon}\right) \in \partial \Omega$ but $X_{\varepsilon}(t) \notin \bar{\Omega}$ for $t \in\left(t_{\varepsilon}, t_{\varepsilon}+\sigma\right)$. Recall that $\left|\beta^{\varepsilon}(t)-\hat{b}_{1}\right|<r$ for $t \in \hat{J}_{1}$. Hence, from $(A 3)$, we see that $X_{\varepsilon}(t) \in \bar{\Omega}$ for $t \in[0, \sigma)$. This contradicts to $(4.3)$.

Therefore, in view of the choice of $\delta_{0}$, we find $s>0$ such that

$$
H\left(X(t), \phi(X(t)), D \phi(X(t)) ; \delta_{0}[\beta](t), \beta(t)\right) \geq \frac{\theta}{2}
$$

for $t \in[0, s]$ and $\beta \in \mathcal{B}_{s}(x)$. Multiplying this inequality with $e^{-\lambda t}$ and then integrating that over $(0, s)$, we conclude the proof.

We note that the proof above of (ii) is more delicate than that of (i) since we have to construct an "admissible" strategy $\delta_{0} \in \Delta_{s}(x)$ in the proof of (ii). We refer to [6] for the original idea.

We can obtain similar results to the lemma above for the upper Hamiltonians.

Lemma 4.2 Assume $(A 1)$.

(1) Let $(A 2)$ also hold. For any $\theta>0$ and $\phi \in C^{1}(\bar{\Omega})$, there exists $s>0$ such that, if

$$
\bar{H}(x, \phi(x), D \phi(x)) \geq \theta
$$

holds for $x \in \bar{\Omega}$, then there is $\alpha_{0} \in \mathcal{A}_{s}(x)$ such that

$$
\int_{0}^{t} e^{-\lambda r} H\left(X_{\gamma}(r), \phi\left(X_{\gamma}(r)\right), D \phi\left(X_{\gamma}(r)\right) ; \alpha_{0}(r), \gamma\left[\alpha_{0}\right](r)\right) d r \geq \frac{\theta}{2 \lambda}\left(1-e^{-\lambda t}\right)
$$

for $t \in(0, s]$ and $\gamma \in \Gamma_{t}(x)$, where $X_{\gamma}(r)=X\left(r ; x, \alpha_{0}, \gamma\left[\alpha_{0}\right]\right)$.

(2) Let $\left(A 3^{\prime}\right)$ also hold. For any $\theta>0$ and $\phi \in C^{1}(\bar{\Omega})$, there exists $s>0$ such that, if

$$
H_{+}(x, \phi(x), D \phi(x)) \leq-\theta
$$

holds for some $x \in \bar{\Omega}$, then there is $\gamma_{0} \in \Gamma_{s}(x)$ such that

$$
\int_{0}^{t} e^{-\lambda r} H\left(X_{\alpha}(r), \phi\left(X_{\alpha}(r)\right), D \phi\left(X_{\alpha}(r)\right) ; \alpha(r), \gamma_{0}[\alpha](r)\right) d r \leq-\frac{\theta}{2 \lambda}\left(1-e^{-\lambda t}\right)
$$


for $t \in(0, s]$ and $\alpha \in \mathcal{A}_{t}(x)$, where $X_{\alpha}(r)=X\left(r ; x, \alpha, \gamma_{0}[\alpha]\right)$.

5. Viscosity solutions. We shall adapt the following definition of viscosity solutions for the SC problems of (1.1土).

Definition. We call $u$ a subsolution (resp., supersolution and solution) for

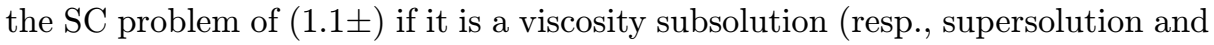
solution) of

$$
H_{ \pm}(x, u(x), D u(x))=0 \text { for } x \in \bar{\Omega} \text {. }
$$

Remarks. We refer to [2] for the definition of viscosity solutions on the closure of $\Omega$.

We note that $H_{-}(x, r, p)=\underline{H}(x, r, p)$ and $H_{+}(x, r, p)=\bar{H}(x, r, p)$ provided $(x, r, p) \in \Omega \times \mathbf{R} \times \mathbf{R}^{n}$.

We remark that $H_{-}^{*}(x, r, p)=\underline{H}(x, r, p)$ and $H_{+*}(x, r, p)=\bar{H}(x, r, p)$ for $(x, r, p) \in \partial \Omega \times \mathbb{R} \times \mathbb{R}^{n}$. Moreover, since the mappings $x \in \bar{\Omega} \mapsto A(x, b) \in 2^{A}$ and $B(x, a) \in 2^{B}$ are lower semicontinuous for any fixed $b \in B$ and $a \in A$, respectively, we see that

$$
H_{-*}(x, r, p)=H_{-}(x, r, p) \text { and } H_{+}^{*}(x, r, p)=H_{+}(x, r, p)
$$

for $x \in \partial \Omega \times \mathbb{R} \times \mathbb{R}^{n}$. Here, lower and upper $*$, respectively, denote the lower and upper semicontinuous envelopes of the original functions.

Theorem 5.1 Assume $(A 1)$ and $(A 3)$ ( resp., $(A 1)$ and $\left(A 3^{\prime}\right)$ ). Then, the lower (resp., upper) value function $V($ resp. $U)$ is a viscosity solution of $(S C-)$ (resp., $(S C+))$.

Proof. We only prove the assertion for $V$ since the other one can be proved with a similar argument below by using Lemma 4.2 and Theorem 3.1 (ii) in place of Lemma 4.1 and Theorem 3.1 (i).

We also only show the assertion for subsolutions. Indeed, the assertion for supersolutions can be shown similarly by utilizing Lemma 4.1 (i) instead of Lemma 4.1 (ii) in the proof below.

Let $\hat{x} \in \bar{\Omega}$ be a maximum point of $V^{*}-\phi$ for some $\phi \in C^{1}$. We may suppose $\hat{x} \in \partial \Omega$ since we can proceed the argument in [6] in case when $\hat{x} \in \Omega$.

Subtracting a constant from $\phi$, we may also suppose that

$$
V^{*}(\hat{x})=\phi(\hat{x}) \text { and } V^{*} \leq \phi \text { in } \bar{\Omega} .
$$

We suppose that there is $\theta>0$ such that

$$
H_{-*}(\hat{x}, \phi(\hat{x}), D \phi(\hat{x})) \geq 2 \theta
$$


and then, we will get a contradiction.

We first observe that, for each $\varepsilon>0$, there is $x_{\varepsilon} \in \bar{\Omega} \cap B_{\varepsilon}(\hat{x})$ such that

$$
\phi\left(x_{\varepsilon}\right)<\varepsilon+V\left(x_{\varepsilon}\right) .
$$

We may suppose $x_{\varepsilon} \in \partial \Omega$ again.

For sufficiently small $\varepsilon>0,(5.2)$ implies

$$
H_{-}\left(x_{\varepsilon}, \phi\left(x_{\varepsilon}\right), D \phi\left(x_{\varepsilon}\right)\right) \geq \theta \text {. }
$$

Using Lemma 4.1 (ii), we find $s>0$ and $\delta_{\varepsilon} \in \Delta_{s}\left(x_{\varepsilon}\right)$ such that

$$
-\int_{0}^{t} e^{-\lambda r} f\left(X_{\beta}(r), \delta_{\varepsilon}[\beta](r), \beta(r)\right) d r+\phi\left(x_{\varepsilon}\right)-e^{-\lambda t} \phi\left(X_{\beta}(t)\right) \geq \frac{\theta}{2 \lambda}\left(1-e^{-\lambda t}\right)
$$

for $t \in(0, s]$ and $\beta \in \mathcal{B}_{t}\left(x_{\varepsilon}\right)$, where $X_{\beta}(r)=X\left(r ; x_{\varepsilon}, \delta_{\varepsilon}[\beta], \beta\right)$.

Fix $t=s / 2$ for instance. Since $s>0$ does not depend on $x_{\varepsilon}$, we can choose $\varepsilon<\theta / 4 \lambda\left(1-e^{-\lambda t}\right)$. Substituting (5.1) and (5.3) into the inequality above, we have

$$
V\left(x_{\varepsilon}\right)-J^{t}\left(x_{\varepsilon}, \delta_{\varepsilon}[\beta], \beta\right)-e^{-\lambda t} V\left(X_{\beta}(t)\right) \geq \frac{\theta}{4 \lambda}\left(1-e^{-\lambda t}\right) .
$$

Taking the infimum over $\beta \in \mathcal{B}_{t}\left(x_{\varepsilon}\right)$ and then, taking the supremum over $\delta \in$ $\Delta_{t}\left(x_{\varepsilon}\right)$ in the above, according to Theorem 3.1 (i), we derive that the left hand side of the inequality above is equal to 0 . This is a contradiction.

6. Comparison and uniqueness results. In this section, we present our comparison results for $(S C \pm)$, which imply that the value function constructed in Section 2 is the unique viscosity solution and that it is continuous.

We first introduce the following subsets for $x \in \bar{\Omega}$ :

$$
G_{-}(x, b)=\operatorname{co}\{g(x, a, b) \mid a \in A(x, b)\} \quad(\text { for } b \in B),
$$

and

$$
G_{+}(x, a)=\operatorname{co}\{g(x, a, b) \mid b \in B(x, a)\} \quad(\text { for } a \in A) .
$$

Here, for $K \subset \mathbb{R}^{n}$, co $K$ denotes its convex hull.

We shall use the following hypotheses concerning about the vector fields. 


$$
\left\{\begin{array}{l}
\text { For } z \in \partial \Omega, \text { there are } \theta \in(0,1), r>0 \text { and } \xi \in S^{n-1} \\
\text { such that } \\
\text { (1) } \bigcup_{0<t<r} B_{t \theta}(x+t \xi) \subset \Omega \text { for } x \in \bar{\Omega} \cap B_{r}(z) \\
\text { (2) } G_{-}(z, b) \cap \bigcup_{t>0} B_{t \theta}(t \xi) \neq \varnothing \text { for } b \in B
\end{array}\right.
$$

and

$$
\begin{aligned}
& \text { For } z \in \partial \Omega \text {, there are } \theta \in(0,1), r>0 \text { and } \xi \in S^{n-1} \\
& \text { such that } \\
& \text { (1) } \bigcup_{0<t<r} B_{t \theta}(x+t \xi) \subset \Omega \text { for } x \in \bar{\Omega} \cap B_{r}(z) \\
& \text { (2) } G_{+}(z, a) \cap \bigcup_{t>0} B_{t \theta}(t \xi) \neq \varnothing \text { for } a \in A \text {. }
\end{aligned}
$$

We give a simple example to clarify the hypotheses above on $g(\cdot, \cdot, \cdot)$ near the boundary point where the boundary $\partial \Omega$ is not smooth:

Example. Let $\Omega \subset \mathbb{R}^{2}$ be a bounded open set such that

$$
\Omega \cap B_{1}(0)=\Omega \cap\left\{x=\left(x_{1}, x_{2}\right) \in B_{1}(0) \mid 0<x_{1}, x_{2}<1\right\} .
$$

$A=\left\{a_{i} \mid i=1,2,3\right\}$ and $B=\left\{b_{i} \mid i=1,2\right\}$. Consider the following vector fields:

$$
\begin{aligned}
& g\left(x, a_{i}, b_{1}\right)= \begin{cases}(1,1) & \text { for } i=1 \\
(-1,1) & \text { for } i=2 \\
(1,-1) & \text { for } i=3\end{cases} \\
& g\left(x, a_{i}, b_{2}\right)= \begin{cases}(1,0) & \text { for } i=1 \\
(0,1) & \text { for } i=2 \\
(0,-1) & \text { for } i=3\end{cases}
\end{aligned}
$$

We note that $A\left(0, b_{1}\right)=\left\{a_{1}\right\}$ and $A\left(0, b_{2}\right)=\left\{a_{1}, a_{2}\right\}$. It is easy to check that $(A 4)$ (and $(A 1)-(A 3),(A 5))$ holds at $0 \in \partial \Omega$.

We remark that, for $b=b_{2}$, if we require that one of vectors $g\left(0, a_{i}, b_{2}\right)$ $\left(a_{i} \in A\left(0, b_{2}\right)\right)$ must direct strictly inside of $\Omega$ (in other words, if we replace $G_{-}(z, b)$ in (A4)-(ii) into $\left.\{g(z, a, b) \mid a \in A(z, b)\}\right)$, then we can not treat this example since $g\left(0, a_{i}, b_{2}\right)(i=1,2)$ are tangential at 0 . However, assumption $(A 4)$ allows us to treat this example since a convex combination of those vector fields directs strictly inside of $\Omega$. 
We shall also suppose that the convex combinations of the vector fields appearing in our boundary condition are nondegenerate on $\partial \Omega$;

$$
\inf _{x \in \partial \Omega, b \in B}\left\{|\eta| \mid \eta \in G_{-}(x, b)\right\}>0,
$$

and

$$
\inf _{x \in \partial \Omega, a \in A}\left\{|\eta| \mid \eta \in G_{+}(x, a)\right\}>0 .
$$

We first present a key lemma, which we will prove in the final section:

Lemma 6.1 (cf. [8]) Let $z \in \partial \Omega, r>0, \xi \in S^{n-1}$ and $\theta \in(0,1)$ satisfy that

$$
\bigcup_{0<t<r} B_{t \theta}(x+t \xi) \subset \Omega \text { for } x \in \bar{\Omega} \cap B_{r}(z) .
$$

Then, there are constants $C_{0}, C_{1} \geq 1, \sigma \in(0,1-\theta)$ and a function $\psi \in C^{1}(\bar{\Omega} \times \bar{\Omega})$ such that, for $x, y \in \bar{\Omega} \cap B_{r}(z)$,

$$
\begin{gathered}
C_{0}^{-1}|x-y|^{2} \leq \psi(x, y) \leq C_{0}|x-y|^{2}, \\
\left\langle\xi^{\prime}, D_{x} \psi(x, y)\right\rangle \leq 0 \text { provided } x \in \partial \Omega \text { and } \xi^{\prime} \in B_{\theta+\sigma}(\xi), \\
\left|D_{x} \psi(x, y)\right| \leq C_{1}|x-y| \text { and } D_{x} \psi(x, y)+D_{y} \psi(x, y)=0 .
\end{gathered}
$$

Remark. We note that (6.2) is a stronger requirement than that of [8].

Theorem 6.2 Assume (A1), (A3), (A4), (A5) (resp., (A1), $\left(A 3^{\prime}\right),\left(A 4^{\prime}\right)$, $\left.\left(A 5^{\prime}\right)\right)$. Let $u$ and $v$ be a viscosity subsolution and a viscosity supersolution of $(S C-)($ resp., $(S C+))$, respectively. Then, $u^{*} \leq v_{*}$ in $\bar{\Omega}$.

Proof. We shall show our assertion for $(S C-)$. For simplicity, we shall write $u$ and $v$ instead of $u^{*}$ and $v_{*}$, respectively.

The proof is by contradiction. Suppose $\max _{x \in \bar{\Omega}}\{u(x)-v(x)\} \equiv \Theta>0$. Let $z \in \bar{\Omega}$ be the maximum point; $u(z)-v(z)=\Theta$. We may suppose $z \in \partial \Omega$ since it is rather standard to get a contradiction in case when $z \in \Omega$. (See also the proof below.) We may also suppose that $z$ is the unique maximum point. Indeed, otherwise, we can proceed the argument below by adding a small linear perturbation with no major change. For the details, we refer to [9] for instance. 
Choose the $C^{1}$-function $\psi$ from Lemma 6.1 for the $z$. For $\alpha, \mu>0$, we set

$$
\Psi_{\alpha}(x, y)=u(x)-v(y)-\alpha \psi(x, y)+\mu\langle\xi, x-y\rangle,
$$

where $\mu>0$ will be fixed later and $\alpha>0$ will be sent to $\infty$. Let $\left(x_{\alpha}, y_{\alpha}\right)$ satisfy

$$
\Psi_{\alpha}\left(x_{\alpha}, y_{\alpha}\right)=\max _{x, y \in \bar{\Omega}} \Psi_{\alpha}(x, y) \geq \Theta .
$$

By a standard observation using $\Psi_{\alpha}\left(x_{\alpha}, y_{\alpha}\right) \geq \Psi_{\alpha}(z, z)$ together with (6.1), we have

$$
\lim _{\alpha \rightarrow \infty} x_{\alpha}=\lim _{\alpha \rightarrow \infty} y_{\alpha}=z, \lim _{\alpha \rightarrow \infty} u\left(x_{\alpha}\right)=u(z), \lim _{\alpha \rightarrow \infty} v\left(y_{\alpha}\right)=v(z),
$$

and

$$
\lim _{\alpha \rightarrow \infty} \alpha\left|x_{\alpha}-y_{\alpha}\right|^{2}=0 .
$$

For simplicity, we shall write $x$ and $y$ in place of $x_{\alpha}$ and $y_{\alpha}$, respectively.

We will show that the following inequalities hold for large $\alpha>0$ and for a fixed $\mu>0$.

$$
\underline{H}\left(x, u(x), \alpha D_{x} \psi(x, y)-\mu \xi\right) \leq 0 \leq \underline{H}\left(y, v(y),-\alpha D_{y} \psi(y, y)-\mu \xi\right) .
$$

In fact, once we obtain (6.6), we easily get a contradiction. Indeed, using (6.3) and $(A 1)$, by $(6.6)$, we have

$$
\lambda(u(x)-v(y)) \leq C|x-y|(1+\alpha|x-y|)
$$

for some $C>0$ independent of $\alpha>0$. Hence, sending $\alpha \rightarrow \infty$ in (6.7) together with (6.4) and (6.5), we have $\lambda \Theta \leq 0$. This is a contradiction.

Now we shall concentrate our attention to derive (6.6). Notice that the second inequality in (6.6) always holds in this case. Let us suppose that the first inequality in (6.6) fails;

$$
\underline{H}\left(x, u(x), \alpha D_{x} \psi(x, y)-\mu \xi\right)>0 .
$$

This yields that $x \in \partial \Omega$. Then, it follows

$$
H_{-}\left(x, u(x), \alpha D_{x} \psi(x, y)-\mu \xi\right) \leq 0 .
$$

Thus, there is $\hat{b} \in B$, for some $l \in \mathbf{N}$, such that

$$
H\left(x, u(x), \alpha D_{x} \psi(x, y)-\mu \xi ; a, \hat{b}\right) \leq 0
$$


for all $a \in A(x, \hat{b})$. In view of $(A 4)$-(ii), we can choose $\left\{t_{k}>0\right\}_{k=1}^{\ell}$ and $\left\{a_{k} \in\right.$ $A(z, \hat{b})\}_{k=1}^{\ell}$ such that

$$
\sum_{k=1}^{\ell} t_{k}=1 \text { and } \sum_{k=1}^{\ell} t_{k} g\left(z, a_{k}, \hat{b}\right) \in \bigcup_{t>0} B_{t \theta}(t \xi)
$$

Set $\eta(x) \equiv \sum_{k=1}^{\ell} t_{k} g\left(x, a_{k}, \hat{b}\right)$. Taking a convex combination over $a \in A(z, \hat{b})(\subset$ $A(x, \hat{b}))$ in the inequality above, we have

$$
-\left\langle\eta(x), \alpha D_{x} \psi(x, y)-\mu \xi\right\rangle \leq \lambda\|u\|_{L^{\infty}(\Omega)}+\max _{(a, b) \in A \times B}\|f(\cdot, a, b)\|_{L^{\infty}(\Omega)} .
$$

In view of $(A 1)-(\mathrm{iii})$ and $(A 5)$, there is a contant $k \in\left[K_{1}, K_{2}\right]$ such that $k \eta(z) \in$ $B_{\theta}(\xi)$, where

$$
\begin{aligned}
& K_{1} \equiv \inf _{x \in \partial \Omega, b \in B}\left\{\frac{1}{|\eta|} \mid \eta \in G_{-}(x, b)\right\}, \\
& K_{2} \equiv \sup _{x \in \partial \Omega, b \in B}\left\{\frac{1}{|\eta|} \mid \eta \in G_{-}(x, b)\right\} .
\end{aligned}
$$

By (6.4), for large $\alpha>1$, we see that $k \eta(x) \in B_{\theta+\sigma}(\xi)$. Hence, by (6.2) and (6.8), we have

$$
\mu k|\eta(x)| \sqrt{1-(\theta+\sigma)^{2}} \leq C
$$

for some $C>0$. Therefore, we get a contradiction for a large $\mu>1$.

In order to show the assertion for $(S C+)$, we can proceed a similar argument to that for $(S C-)$ by taking the following $\Psi_{\alpha}$ in place of that in the above:

$$
\Psi_{\alpha}(x, y)=u(x)-v(y)-\alpha \psi(y, x)-\mu\langle\xi, x-y\rangle .
$$

Now, according to Theorem 6.2 , it is easy to show the uniqueness and continuity of the lower and upper value functions.

Corollary 6.3 Assume $(A 1),(A 3),(A 4),(A 5)$ (resp., $(A 1),\left(A 3^{\prime}\right),\left(A 4^{\prime}\right)$, $\left.\left(A 5^{\prime}\right)\right)$. Then, $V$ ( resp., $\left.U\right)$ is the unique viscosity solution of $(S C-)$ ( resp., $(S C+))$. Moreover, $V \in C(\bar{\Omega})($ resp., $U \in C(\bar{\Omega}))$.

7. A sketch of proof of Lemma 6.1. By translation we may suppose $z=0 \in \partial \Omega$. Let $\xi \in S^{n-1}, r>0$ and $\theta \in(0,1)$ satisfy

$$
\bigcup_{0<t<r} B_{t \theta}(x+t \xi) \subset \Omega \text { for } x \in \bar{\Omega} \cap B_{r}(0),
$$


and

$$
\bigcup_{0<t<r} B_{t \theta}(x-t \xi) \subset \bar{\Omega}^{c} \text { for } x \in \Omega^{c} \cap B_{r}(0) .
$$

We may also suppose that there is $\sigma>0$ such that

$$
\bigcup_{0<t<r} B_{t(\theta+\sigma)}(x+t \xi) \subset \Omega \text { for } x \in \bar{\Omega} \cap B_{r}(0)
$$

and

$$
\bigcup_{0<t<r} B_{t(\theta+2 \sigma)}(x-t \xi) \subset \bar{\Omega}^{c} \text { for } x \in \partial \Omega \cap B_{r}(0) .
$$

We introduce the following closed convex sets

$$
L \equiv \bigcup_{t \geq 0} B_{t(\theta+2 \sigma)}(t \xi), K \equiv \bigcup_{t \geq 0} B_{t(\theta+\sigma)}(t \xi), K_{0}=\bigcup_{0 \leq t \leq r} B_{t(\theta+\sigma)}(t \xi)
$$

and

$$
K_{\varepsilon} \equiv\left\{x \in \mathbb{R}^{n} \mid \operatorname{dist}\left(x, K_{0}\right) \leq \varepsilon\right\} .
$$

We choose a small $\varepsilon>0$ so that

$$
\partial K_{\varepsilon} \backslash L=\left\{x \in \mathbb{R}^{n} \backslash L \mid \operatorname{dist}(x, K)=\varepsilon\right\} .
$$

We define the Minkowskii functional $v$ associated with $K_{\varepsilon}$;

$$
v(x)=\inf \left\{\alpha>0 \mid x \in \alpha K_{\varepsilon}\right\} .
$$

From the definition, we see that $v(t x)=t v(x)$ for $t>0$ and $x \in \mathbb{R}^{n} \backslash\{0\}$ and that $v(x)=v(-x)$ for $x \in \mathbb{R}^{n} \backslash\{0\}$. Since 0 is an interior point of $K_{\varepsilon}$, we see that there is $C \geq 1$ such that

$$
C^{-1}|x| \leq v(x) \leq C|x| .
$$

We define $\psi(x, y)=v^{2}(x-y)$. This definition together with the above inequalities yields that (6.1) and (6.3) hold.

To show (6.2), we fix $\xi^{\prime} \in B_{\theta+\sigma}(\xi), x \in \partial \Omega \cap B_{r}(0)$ and $y \in \bar{\Omega} \cap B_{r}(0)$. By (7.1), it suffices to show that

$$
\left\langle\xi^{\prime}, D_{x} v(x-y)\right\rangle \leq 0 \text { whenever } x-y \notin L .
$$


We may also suppose that $x-y \in \partial K_{\varepsilon} \backslash L$ because of the homogeneity of $v$.

We remark that $v\left(x-y+t \xi^{\prime}\right) \leq 1$ for small $t>0$ because $x-y+t \xi^{\prime} \in K_{\varepsilon}$ for small $t>0$. Thus, we have

$$
\lim _{t \downarrow 0} \frac{v\left(x-y+t \xi^{\prime}\right)-v(x-y)}{t} \leq 0 .
$$

Acknowledgement. The author would like to thank the referee for several suggestions for the first draft.

\section{REFERENCES}

[1] I. Capuzzo-Dolcetta and P.-L. Lions, Hamilton-Jacobi equations with state constraints, Trans. Amer. Math. Soc., 318 (1990), 643-684.

[2] M. G. Crandall, H. Ishi and P.-L. Lions, User's guide to viscosity solutions of second order partial differential equations, Bull. Amer. Math. Soc., 27 (1992), 1-67.

[3] P. Dupuis And H. Ishis, On oblique derivative problems for fully nonlinear second-order elliptic partial differential equations on nonsmooth domains, Nonlinear Anal., 15 (1990), 1123-1138.

[4] L. C. Evans and H. IshiI, Differential games and nonlinear first order PDE on bounded domains, Manuscripta Math., 49 (1984), 109-139.

[5] R. Elliott and N. Kalton, Boundary value problems for nonlinear partial differential equations, J. Math. Anal. Appl., 46 (1974), 228-241.

[6] L. C. Evans and P. E. Souganidis, Differential games and representation formulas for solutions of Hamilton-Jacobi-Isaacs equations, Indiana Univ. Math. J., 33 (1984), 773-797.

[7] H. IshiI, A boundary value problem of the Dirichlet type for Hamilton-Jacobi equations, Ann. Scoula Norm. Sup. Pisa, 16 (1989), 14-45.

[8] H. Ishi and S. KoIKe, A new formulation of state constraints problem of first-order PDEs, to appear in SIAM J. Control \& Optim.

[9] H. Ishir and S. KoIKe, Viscosity solutions of a system of nonlinear secondorder elliptic PDEs arising in switching games, Funkcial. Ekvac., 34 (1991), $143-155$.

[10] M. Katsoulakis, Viscosity solutions of 2nd order fully nonlinear elliptic equations with state constraints, Indiana Univ. Math. J., 43 (1994), 493-519.

[11] M. Katsoulakis and S. KoIKe, viscosity solutions of monotone systems for Dirichlet problems, Differential Integral Equations, 7 (1994), 367-382.

[12] S. KoIкE, Uniqueness of viscosity solutions for monotone systems of fully nonlinear PDEs under Dirichlet condition, Nonlinear Anal., 22 (1994), 519532.

[13] P.-L. Lions and P. Souganidis, Differential games, optimal control and directional derivatives of viscosity solutions of Bellman's and Isaacs' equations, SIAM J. Control \& Optim., 23 (1985), 566-583.

[14] M. H. Soner, Optimal control with state-space constraint I, SIAM J. Control \& Optim., 24 (1986), 552-562.

[15] D. Tataru, Boundary value problems for first order Hamilton-Jacobi equations, Nonlinear Anal., 19 (1992), 1091-1110.

Supported in part by Grant-in-Aid for Scientific Research (No. 05640151) of the Ministry of Education, Science and Culture. 
Department of Mathematics

Saitama University

255 Shimo-Okubo, Urawa

Saitama 338, Japan

Received: August 5th, 1994; revised: February 16th, 1995. 